\title{
Rodent models of sepsis found shockingly lacking
}

When Kevin Tracey and his colleagues at the Feinstein Institute for Medical Research in Manhasset, New York, set out to test whether they could reverse the cognitive impairments associated with sepsis, they naturally turned to the mouse model of cecal ligation and puncture (CLP). This method of introducing a microbial infection into mice is generally considered the go-to preclinical model in sepsis research. Reporting in May, Tracey's team showed that they could improve memory defects in the CLP model by blocking a protein called high mobility group box-1 (HMGB1), a key mediator of the disease (Mol. Med. doi:10.2119/ molmed.2012.00195, 2012). But this gain might be a red herring, as rodents remain a poor stand-in for severe sepsis in people. "The mouse models really don't reflect the human condition," says Shaw Warren, an infectious disease specialist at the Massachusetts General Hospital in Boston.

The CLP model was developed more than 30 years ago as a more clinically realistic way to induce sepsis in rodents than previous techniques, which relied mostly on injecting purified bacteria or their toxins into rodents. Using the CLP model-which involves perforating the large intestine to allow fecal material into the peritoneal cavity, thereby triggering the inflammatory immune response typically seen in human sepsis-researchers can tailor the severity of the disease by manipulating the size and timing of the gut breach. But the technique still misses the mark for several reasons.

For one, researchers usually use young, healthy, inbred mice that die fairly quickly after disease onset. "This is very different from the human situation," notes Tracey. "Death and other end points [in humans] develop over weeks and months." The CLP method is also highly prone to experimental error, as factors such as the length of intestine tied off, the size of the needle or the amount of fecal material released can lead to major differences in disease outcome. Peter Ward and his colleagues at the University of Michigan Medical School in Ann Arbor have tried to overcome some of these issues by proposing a set of standardized procedures for inducing sepsis in mice and rats (Nat. Protoc. 4, 31-36, 2009).

Still, variability problems persist. "The unspoken truth is that when identical insults are given to identically aged groups of animals-even from the same litter-we see a variable individual effect," says Mervin Singer, an intensive-care specialist who studies mouse models of sepsis at University College London.

\section{Comorbid curiosity}

Some in the field are developing new ways to fine-tune the induction system so that it better mimics the poor health that many sick people are in when they develop sepsis. For example, Robert Star and his colleagues at the US National Institute of Diabetes and Digestive and Kidney Diseases in Bethesda, Maryland, recently stimulated chronic kidney disease in mice before they proceeded with traditional CLP to provoke sepsis. In a study published last year, they showed that the kidney disease worsened the sepsis severity and sepsis-induced organ damage seen in the mice, as evidenced by increased blood levels of HMGB1, vascular endothelial growth factor and other inflammatory cytokines (Kidney Int. 80, 1198-1211, 2011).

"Animal models have to mimic the epidemiologic causes [of sepsis]," Star says. "You have to replicate whatever you have found in humans."

In the end, though, most experts agree that tweaks like these are just minor improvements and that the field really needs to radically redesign animal models of sepsis from the ground up. "Clearly, current animal models seem to be incapable of predicting results in human trials of new agents," says Mitchell Fink, a surgeon at the University of California-Los Angeles. Alas, until they do, few drug developers will be willing to move forward with a drug like Tracey's antibody to HMGB1, despite the promising preclinical results.

Kathleen Raven

\section{Execution of sepsis trials needs an overhaul, experts say}

The search for new sepsis drugs continues apace, but now, more than ever, experts say that overlooking the heterogeneous nature of this condition will derail any hopes of proving that these therapies work under the strict scrutiny of a large-scale, randomized clinical trial. "It's exhausting to watch drug companies come out with a compound that they are excited about and then evaluate it in a way that was done in the late 1980s," says Derek Angus, a critical-care specialist at the University of Pittsburgh School of Medicine in Pennsylvania.

The problems begin with study endpoints. In a commentary published late last year, Angus points out that many patients remain hospitalized beyond the 28-day period typically used as the primary clinical endpoint in phase 3 trials (JAMA 306, 2614-2615, 2011). He suggests that trials should focus on a larger window of time and perhaps even shift the focus of drug development in sepsis toward manipulating the patient's immune response further out in time than just a month.

Angus is not alone in calling for this type of rethink. "A number of recent trials have shown that mortality signals are more stable at longer time points-for example, 60 or 90 days," says John Marshall, a critical-care surgeon at St. Michael's Hospital in Toronto. He cites a 2009 study he coauthored that found more steady survival rates three months out in critically ill patients in Australia and Canada (N. Engl. J. Med. 360, 1283-1297, 2009).

Researchers also caution against lumping patients with sepsis into trials without taking into account the fact that causes of the condition vary. Most cases of sepsis are caused by bacteria, but some cases have fungi to blame and some don't have an apparent pathogenic trigger at all. "In the 1980s, we were convinced sepsis was related to infection and infection only," says Paul Walker, chief executive of Spectral Diagnostics, a Toronto-based company developing sepsis-related products. But now, researchers have documented septic shock in individuals following a massive trauma, such as that sustained in a car accident, with no detectable foreign pathogen as the root cause.

Walker's company is currently funding a phase 3 trial in which its diagnostic tool will help identify and stratify study participants according to amount of endotoxin circulating in their system 